\title{
A model for ink impression into a porous substrate
}

\begin{abstract}
This paper applies the physics of a squeezed film between porous surfaces to the impression of an ink dot into paper. The models developed are implemented numerically via the finiteelement method. When compared with observations reported in the literature, the models are shown to be capable of representing the effects of process parameters such as the pressure, viscosity, permeability and film thickness on the dot gain which is used frequently as a characteristic to measure printing quality.
\end{abstract}

Keyword: Squeezed film; Ink dot; Porous surfaces 